Original Research

\title{
Differential Expression of Monocarboxylate Transporter 1 and Ancillary Protein CD147 in Red Blood Cells of Show Jumping Horses
}

\author{
Walter Heinz Feringer Júnior ${ }^{a}$, Julia Ribeiro Garcia de Carvalho ${ }^{a}$, \\ Maria Luiza Mendes de Almeida ${ }^{\mathrm{b}}$, Eliana Gertrudes Macedo Lemos ${ }^{\mathrm{b}}$, \\ Otávio Augusto Brioschi Soares ${ }^{\mathrm{c}}$, Gesiane Ribeiro ${ }^{\mathrm{d}}$, Antonio de Queiroz-Neto ${ }^{\mathrm{a}}$, \\ Guilherme de Camargo Ferraz ${ }^{\text {a,* }}$
}

${ }^{a}$ Faculdade de Ciências Agrárias e Veterinárias, UNESP Univ Estadual Paulista, Campus de Jaboticabal, Departamento de Morfologia e Fisiologia Animal, Laboratório de Farmacologia e Fisiologia do Exercício Equino (LAFEQ) Jaboticabal, São Paulo, Brazil

${ }^{\mathrm{b}}$ Faculdade de Ciências Agrárias e Veterinárias, UNESP Univ Estadual Paulista, Campus de Jaboticabal, Departamento de Tecnologia, São Paulo, Brazil

${ }^{\mathrm{c}}$ Brazilian Army, São Paulo, Brazil

${ }^{\mathrm{d}}$ Faculdades Metropolitanas Unidas, HOVET, São Paulo, Brazil

\section{A R T I C L E I N F O}

\section{Article history:}

Received 4 July 2016

Received in revised form 10 November 2016

Accepted 13 November 2016

Available online 20 November 2016

\section{Keywords:}

Lactate transporter complex

MCT1

CD 147

Lactate

Exercise physiology

\begin{abstract}
A B S T R A C T
We compare the expression levels of the lactate transporter complex consisting of the lactate transporter, monocarboxylate transporter 1 (MCT1), and its ancillary protein, cluster of differentiation 147 (CD147), in the membranes of red blood cells (RBCs) from two breeds of jumping horses and associate the expression levels of these proteins with their jumping ability. The expression levels of MCT1 and CD147 proteins on the membranes of RBCs collected from 30 show jumping horses of two different breeds were quantified: the Brazilian Sport Horses $(n=17)$ and the European Warmbloods $(n=13)$. The levels of MCT1 and CD147 in the RBC membranes were measured by western blot using horse-specific antibodies. Statistical analyses included unpaired Student $t$ test and chi-squared test. According to the expression levels of MCT1 and CD147 proteins, 88\% of the Brazilian Sport Horses were categorized as high lactate transporters (HTs) and the remaining $12 \%$ as low lactate transporters (LTs). The opposite was found for the European Warmbloods, where most animals (77\%) were classified as LTs and the remaining animals (23\%) were classified as HTs. Brazilian Sport Horses express statistically significantly higher levels of CD147 and MCT1 than European Warmbloods. The classification of horses considering the expression of proteins involved in the ability to transport lactate through the complex MCT1-CD147 seems to be breed dependent, with horses that are able to jump higher obstacles showing lower expression of the MCT1-CD147 complex in their RBCs.
\end{abstract}

(C) 2016 Elsevier Inc. All rights reserved.

\footnotetext{
* Corresponding author at: Guilherme de Camargo Ferraz, UNESP - Univ Estadual Paulista, Departamento de Morfologia e Fisiologia Animal, São Paulo, Jaboticabal, Brazil.

E-mail address: gferraz@fcav.unesp.br (G. de Camargo Ferraz).
}

\section{Introduction}

During intense exercise, glycolysis contributes to energy production with a concomitant increase in the concentration of muscle lactate and $\mathrm{H}^{+}$ions. These ions are carried simultaneously from the myofibers onto the circulation (blood/plasma) by monocarboxylate transporter (MCT). This mechanism may create a lactate concentration 
gradient between the muscular compartment and the plasma, favoring the removal of lactate and $\mathrm{H}^{+}$ions from the muscle, thereby affecting intramuscular $\mathrm{pH}$ during exercise [1]. Among the $14 \mathrm{MCT}$ isoforms identified so far, MCT1 is the best studied [2] and the most abundant in equine red blood cells (RBCs) membranes [1]. This protein requires the ancillary protein cluster of differentiation 147 (CD147) [3] to enable to translocate lactate and $\mathrm{H}^{+}$ions from the cytoplasm to the plasma membrane [4]. In horses, up to $50 \%$ of these ions can be carried into RBCs [5]. According to Mykkänen et al [1], lactate transport activity can be correlated to the optical density (OD) of the MCT1-CD147 complex in the RBC's membranes of horses.

Studies examining Standardbred horses stated that MCT1 and CD147 expression is distributed bimodally in high lactate transporters (HTs) and low lactate transporters (LTs), lactate transport activity in RBCs [5,6], and extended to Thoroughbred and Finnhorses [1]. In the latter study [1], three different breeds were compared, including specificities (Trotters vs. Racehorses) and different athletic abilities. Furthermore, training has been shown to increase RBC lactate transport activity in reindeer and sled dogs, but not in horses $[1,6]$.

The levels of MCT1 and CD147 mRNA correlated with the expression of CD147 and suggest that the bimodality of their expression is regulated at the transcriptional level [7]. However, little is known about the expression profiles of the MCT1 and CD147 proteins in jumping horses. The present study is the first to investigate the expression profiles of MCT1 and CD147 in jumping horses and to associate the expression patterns with the jumping ability of the animals.

\section{Materials and Methods}

\subsection{Horses}

Thirty horses were distributed into two groups according to their breeds: group Brazilian Sports Horses ( $\mathrm{BH}, \mathrm{n}=$ 17; body weight $490 \pm 53 \mathrm{~kg}$; age $11 \pm 3$ years) and group European Warmbloods (EW, $\mathrm{n}=13$; body weight $550 \pm$ $50 \mathrm{~kg}$; age $10 \pm 4$ years). The $\mathrm{BH}$ horses belong to the Brazilian Army and are stabled in Resende, RJ, Brazil. They were also used in a recently published study [8]. The EW horses belong to the Brazilian elite Equestrian Society (Sociedade Hípica Paulista).

At the time of blood sampling, all horses had participated in jumping competitions with height obstacles no higher than $1.20 \mathrm{~m}$ for the $\mathrm{BH}$ group and no lower than $1.30 \mathrm{~m}$ for the EW group. The horses were clinically healthy and all had participated for at least 10 months. The study followed the Ethical Principles in Animal Experimentation (Jaboticabal, Brazil, Approval number: 019 281/13). The results have been partially presented at the ninth International Conference on Equine Exercise Physiology (ICEEP 9) in June 2014, in Chester, England [9].

\subsection{Collection of Blood Samples and Western Blot}

Venous jugular blood samples were collected, at rest, in a tube containing ethylenediaminetetraacetic acid, and used to isolate membranes from RBCs according to the method described by Koho et al [5]. Briefly, excess hemoglobin was removed by washing three times with $35 \mathrm{~mL}$ of $5 \mathrm{mM}$ sodium phosphate buffer (PBS) pH 8.0 followed by centrifugation at $20,000 \times g$ and $4^{\circ} \mathrm{C}$ for 15 minutes to separate the RBC membranes from the total cell lysate. The samples were then resuspended in $100 \mathrm{~mL}$ of the phosphate buffer. The protein concentration was determined using the bicinchoninic acid (BCA) method (Pierce BCA Protein Assay Kit, Thermo Fisher Scientific, Waltham, MA).

For the Western blotting, the membranes containing $40 \mu \mathrm{g}$ of protein were solubilized in Laemmli sample buffer, separated on a 10\% (w/v) SDS-PAGE gel (125 vs. $90 \mathrm{mi}-$ nutes), and electro-transferred ( $100 \mathrm{~V}, 60$ minutes) onto nitrocellulose membranes (Protran). Nonspecific binding sites were blocked with $5 \%$ bovine serum albumin (BSA) in $1 \times$ TBST supplemented with $0.1 \%$ Tween 20 .

The membranes were incubated in $1 \%$ BSA in YBST overnight at $4^{\circ} \mathrm{C}$ with horse anti-CD147 or anti-MCT1 primary antibodies against the C-termini of horse MCT1 and CD147 (sequences CKGTEGDPKEESP and CGHHVNDKDKNVRQRNAS, GenBank accession no. AAR21622.1 and ABQ53583.1, respectively). These antibodies were raised in rabbits and purified by affinity chromatography. They were kindly provided by the Nina Koho of Helsinki University.

The membranes were subsequently incubated with peroxidase-conjugated antirabbit antibody (DAKO). The chemiluminescence capture system (ChemiDoc MP-Bio-Rad) with developer (Bio-Rad) was used for visualization. The reference sample was from a known horse with a high amount of CD147, whose OD value was adjusted to 1.0. Thus, the OD of each experimental immunoblot and, therefore, the average amount of CD147 from each group were expressed as the amount of protein relative to the reference sample [10]. The bands were quantified using the Image Lab (Bio-Rad 4.0) software. The bimodal expression was determined by the CD147 amount according to the method described by Mykkänen et al [1]. The categorization of samples from the horse breeds as LT or HT was based on the ODs obtained in the Western blot experiment.

\subsection{Statistics}

Differences between breeds were determined using the chi-square test and unpaired Student $t$ test. The level of significance was set at $P<.05$. All calculations were performed using the R statistical analysis software.

\section{Results}

Bands corresponding to the expected molecular weights of MCT1 (44 kDa) and CD147 (46 kDa) were detected in samples from both breeds of jumping horses analyzed (Fig. 1). However, the expression levels of both proteins were lower $(P<.01)$ in EW horses than in BH horses (Fig. 2). In the $\mathrm{BH}$ breed, $88 \%$ and $12 \%$ of the horses were classified as HT and LT, respectively. The opposite was seen in the EW breed with most of the horses (77\%) being classified as LT and $23 \%$ as HT. Fig. 3 shows that the distribution of MCT1 and CD147 in the HT or LT animals is 
$\mathrm{BH}$ EW

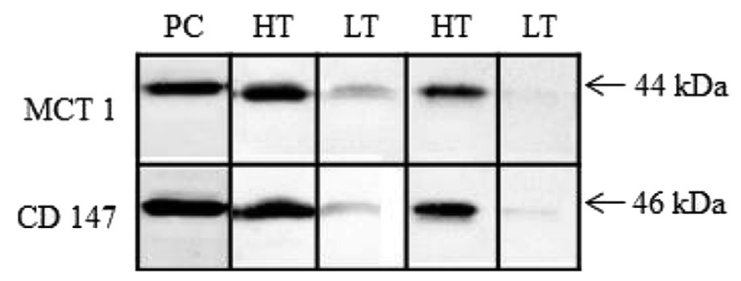

Fig. 1. Western blot detection of CD147 and MCT1 from jumping horses. Note the similar expression levels of the proteins. In most samples of LT horses, the 44 and $46 \mathrm{kDa}$ bands were faint, whereas in most HT horses, the bands showed strong signal at the same molecular weight. $\mathrm{BH}$, Brazilian Sports Horses; CD147, cluster of differentiation 147; EW, European Warmblood horses; HT, high lactate transport; LT, low lactate transporters; MCT1, monocarboxylate transporter 1; PC, positive control.

breed-dependent $(P<.001)$. The frequency distribution of the amount of MCT1 and CD147 in the horses quantified by Western blot is shown in Fig. 4.

\section{Discussion}

The present study investigates the levels of MCT1 and CD147 expression on the plasma membrane of RBCs from $\mathrm{BH}$ and EW horse breeds, presenting different jumping abilities. We searched for an association between the HT and LT capacities of these horse breeds and the expression levels of MCT1 and CD147.

The present research was a straightforward breed comparison. First, each horse was identified as having a high or low jumping ability. Although this difference may be due in part to the career stage of the horse and different training levels, our study suggests that the distribution of HT and LT horses is breed-dependent. Indeed, the feature HT or LT is congenital, and horses do not change groups with age or training status [11].

A large proportion of Thoroughbreds, trotters, and even the cold-blooded Finnhorses have high lactate transport activity in their RBCs. However, no correlation between the expression of lactate transporters and jumping ability was not detected herein, and the correlation with racing performance was deemed difficult to establish by Mykkänen et al [1].

One aspect that should be considered is that EW horses do not belong to a single breed, but are members of a cluster of saddle horses selected in different European countries out of basic native breeding stocks. This fact may be demonstrated through online searches on sites such as HorseTelex [12], where the activity of each stallion is listed in European associations.

Although numerous exchanges of breeding animals have occurred in Europe, the participation of Thoroughbreds in the genetic make-up of EW has diminished. The EW horses have reached the fixed genetic level of Thoroughbred blood, that is, approximately $40 \%-55 \%$, in most $\mathrm{EW}$, which is the level that works best for current jumping competition. The animals need to be clean, light, have a large gallop stride while being elastic, and have a good mouth and temperament [13]. Indeed, the alleged genetic heterogeneity of Warmbloods may have been compromised by artificial insemination with the widespread use of certain stallions in several countries [14-16].

We found that most of the horses of the EW group were LT (77\%). From a historical perspective, this result may be explained by the long selection period endured by the EW

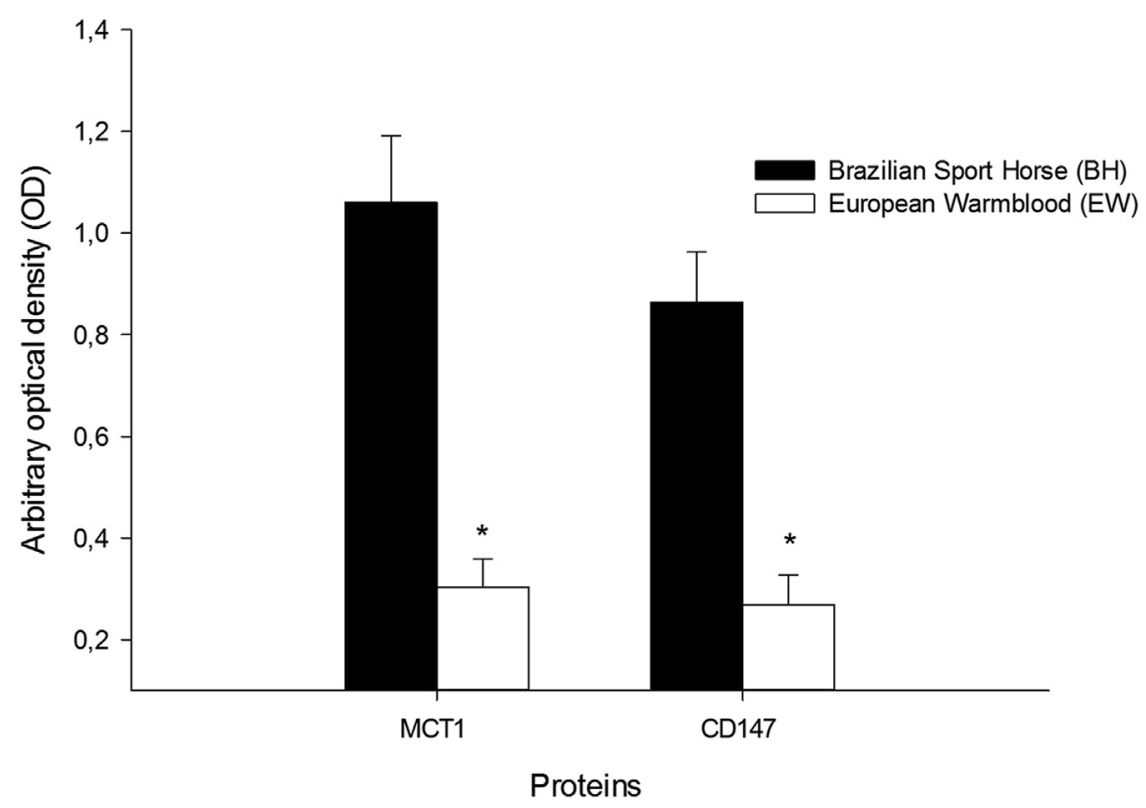

Fig. 2. Mean relative OD values \pm standard deviation for MCT1 and CD147 expression on the membrane of red blood cells of show jumping horses. Statistically significant differences are indicated by * according to the Student $t$ test analysis $(P<.001)$. CD147, cluster of differentiation 147; MCT1, monocarboxylate transporter 1; OD, optical density. 


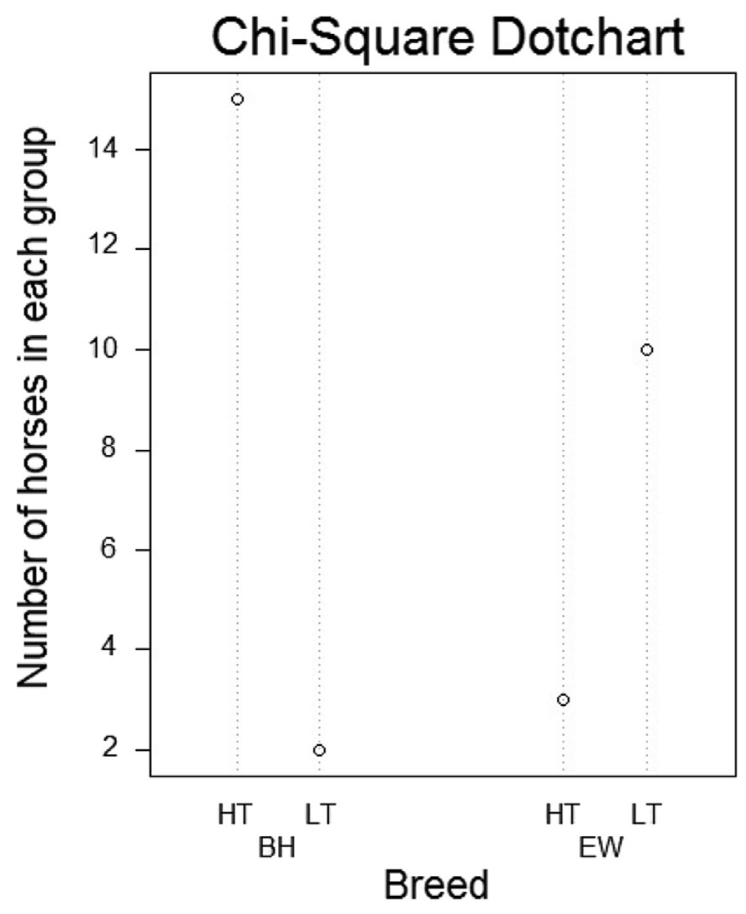

Fig. 3. Dot-chart showing the frequency distribution of horses classified as having HT or LT capacity: BH and EW. There was a difference between the breeds according to the chi-square test $(P<.001)$. BH, Brazilian Sports Horses; EW, European Warmblood horses; HT, high lactate transport; LT, low lactate transporters.

breed during which the jumping ability trait was positively selected at the cost of high speed. Today, the level of Thoroughbred blood is high in almost $90 \%$ of the world's greatest warmblood stallions (approximately 40\%-55\%). Mating these stallions with mares with similar levels of the Thoroughbred breed maintains the level of their blood. For example, mating a mare with $48 \%$ XX with a stallion with $39 \%$ will keep the average at approximately $42 \%-44 \%$ [13].

A great number of the stallions and mares used to breed $\mathrm{BH}$ horses were Thoroughbred racehorses [17]. Following the proposed classification based on OD $[1,5,18]$, we found most of the $\mathrm{BH}$ horses (88\%) were HT, a trait possibly inherited from the Thoroughbreds. The genetic selection of Thoroughbreds caused an increase in the frequency of the C-variant (g.66493737C/T) of the myostatin gene, which contributed to the ability of this breed to race at high speeds [19]. This feature requires a great contribution of muscle anaerobic glycolysis for ATP production, thereby producing substantial amounts of $\mathrm{H}^{+}$and lactate, which are carried by the MCT1-CD147 complex. The BHs studied herein presented an HT population ratio similar to the ones found in other breeds by Mykännen et al [1]: 85\% of HT for Finnhorses; 82\%, for Standardbred; and 88\%, for Thoroughbred.

Indeed, it appears that the breeds of horses that run at high speeds have HT capacity. Our group has quantified the lactate transport complex MCT1-CD147 in the RBC membranes of Quarter Horses and Arabian horses by Western blotting. The intensity of the bands of the MCT1 and CD147 proteins were significantly higher in the membranes of RBCs obtained from Quarter Horses than from Arabian horses [20].

The present study shows that the equine breed EW presents lower expression levels of the proteins that compose the lactate transport complex MCT1-CD147 than $\mathrm{BH}$ animals. Plasma lactate can reach moderate concentrations ( $\sim 5.7 \mathrm{mmol} / \mathrm{L})$ after jumping competitions [21]. There are studies reporting higher lactate concentrations ( $\sim 9.04 \mathrm{mmol} / \mathrm{L})$ [22]. However, these values are still lower than compared with the blood lactate levels found in Thoroughbreds [23].

It has been speculated that the expression intensity of MCT1 and CD147 in the membranes of RBCs may be associated with an increased ability to transport lactate ions from the blood plasma into erythrocytes [5], thereby enhancing the lactate clearing system in horses. The lower blood lactate ion concentration creates a concentration gradient between the intramuscular and plasma lactate ion concentration that stimulates the removal of intramuscular lactate ions [3]. Ultimately, this process may enhance the horse's physical performance due to reduced muscle fatigue [5,24]. Moreover, the bimodal expression of MCT1 and CD147 in the membranes of RBCs is determined genetically, and it is not affected by age, gender, or fitness level [10]. To date, only the expression of muscle MCT1 has been shown to increase its expression levels after a conditioning period [25].

Based on the above information, we hypothesized that horse MCT1 and CD147 expression levels could be directly associated with equine physical performance potential. No such association was observed for the $\mathrm{BH}$ horses, which presented the highest expression of these proteins whereas horses able to jump higher (EW) presented lower amounts of MCT1 and CD147 on the surface of their RBCs. The results obtained for the EW horses were particularly surprising. As noted previously, EW horses are better conditioned against moderate lactatemia. Thus, they probably use oxygen more efficiently, a concept known as "running/jumping" economy defined as the amount of oxygen consumed $(\mathrm{mL} / \mathrm{kg}$ bwt/min) per distance traveled. It is possible that the EW horses consume less oxygen during the execution of jumps at a given speed and height of obstacles, which classifies them as horses with a better jumping economy [26]. In this case, energy metabolism may be strictly aerobic during most of the competition to prevent rapid fatigue due to acidosis. Hence, there is no need to express higher levels of lactate transporters.

This jumping economy may be related to some kinematic variables. The acceleration peaks of the hind limbs at take-off were lower in poor jumpers than in good jumpers. Moreover, the horizontal speed angle was $15^{\circ}$ in successful jumps compared with $12^{\circ}$ in unsuccessful jumps, whereas the vertical components of the velocity were approximately $0.5 \mathrm{~m} /$ second greater in the successfully completed jumps compared with incomplete jumps. Further studies with similar variables, such as age, sex, and kinematic profiles are needed to search for a correlation between lactate transport activity and performance, as well as with the conditioning level [1]. 


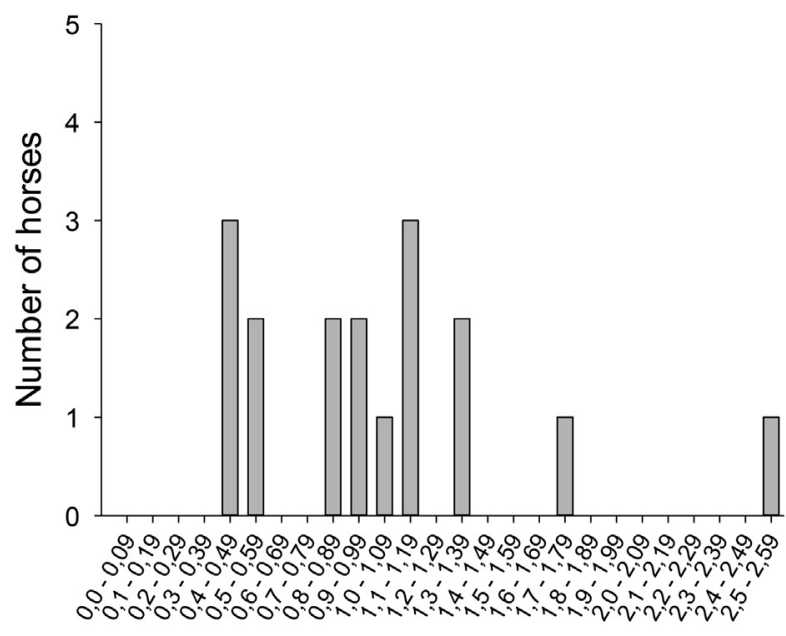

CD $147 \mathrm{BH}$

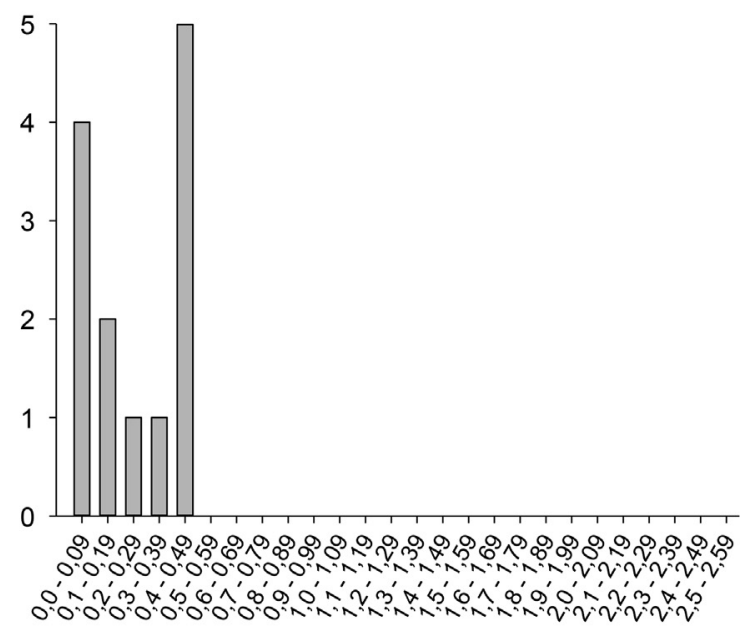

CD 147 EW
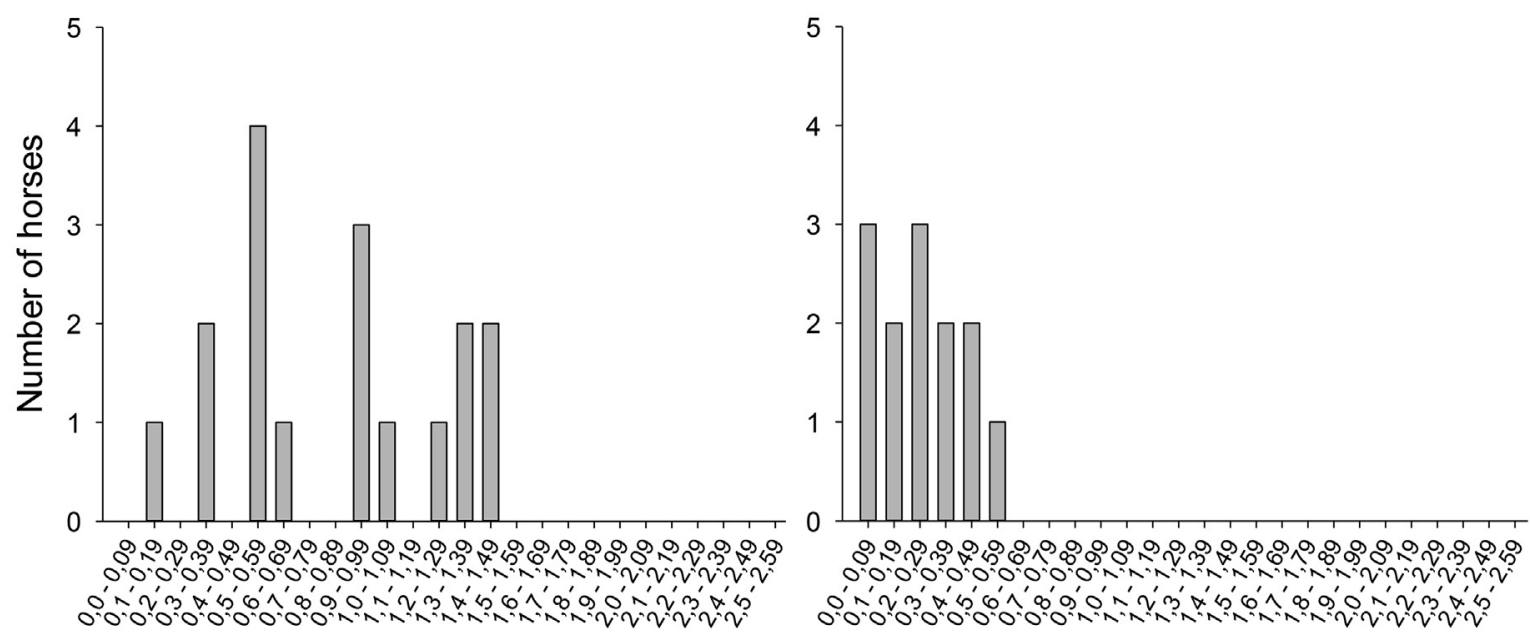

Fig. 4. Frequency distributions of the relative amounts of MCT1 and CD147 as determined by OD evaluation. Western blot samples consisted of erythrocyte (RBC) membranes from two horse breeds: BH and EW. X-axis: relative optical density groups expressed as relative ODs relative to the reference sample, which was arbitrarily set at 1.0; Y-axis: absolute frequency (number) of horses belonging to each OD interval. BH, Brazilian Sports Horses; CD147, cluster of differentiation 147; EW, European Warmblood horses; MCT1, monocarboxylate transporter 1; OD, optical density; RBC, red blood cell.

One limitation of this study is the fact that only protein expression was evaluated. Indeed, high levels of protein expression do not necessarily mean that the transporter is actually functioning more efficiently, in terms of the maximum capacity or affinity of the transporter for its substrate.

This study investigated a subject related to the acid-base homeostasis of show jumping horses [27]. Our results indicated the presence of MCT1 and CD147 in the RBC membranes of the jumping $\mathrm{BH}$ and $\mathrm{EW}$ horse breeds. However, horses of a breed with greater jump ability (EW horses) displayed low lactate transport capacity. The highest lactate carrying capacity was observed in the $\mathrm{BH}$ breed; however, this higher transport capacity does not necessarily translate into a higher jumping ability.

\section{Acknowledgments}

This manuscript was reviewed by a professional science editor and by a native English-speaking copy editor to improve readability. The authors wish to thank the Brazilian Army team for their support in conducting this study and the Fundação de Amparo à Pesquisa do Estado de São Paulo for the financial support (2011/11080-0). The authors are also thankful to Nina Koho for scientific assistance.

None of the authors has financial or personal relationships with people or organizations that could inappropriately influence or bias the content of the study. 


\section{References}

[1] Mykkänen AK, Pösö AR, McGowan CM, McKane SA. Expression of lactate transporters MCT1, MCT2 and CD147 in the red blood cells of three horse breeds: Finnhorse, Standardbred and Thoroughbred. Equine Vet J Suppl 2010;38:161-6.

[2] Halestrap AP. Monocarboxylic acid transport. Compr Physiol 2013; 3:1611-43.

[3] Koho NM, Hyyppä S, Pösö AR. Monocarboxylate transporters (MCT) as lactate carriers in equine muscle and red blood cells. Equine Vet J Suppl 2006;36:354-8.

[4] Gallagher SM, Castorino JJ, Wang D, Philp NJ. Monocarboxylate transporter 4 regulates maturation and trafficking of CD147 to the plasma membrane in the metastatic breast cancer cell line MDAMB-231. Cancer Res 2007;67:4182-9.

[5] Koho NM, Väihkönen LK, Pösö AR. Lactate transport in red blood cells by monocarboxylate transporters. Equine Vet J Suppl 2002;34: 555-9.

[6] Väihkönen LK, Heinonen OJ, Hyyppä S, Nieminen M, Pösö AR. Lactate-transport activity in RBCs of trained and untrained individuals from four racing species. Am J Physiol Regul Integr Comp Physiol 2001;28:R19-24.

[7] Koho NM, Mykkänen AK, Reeben M, Raekallio MR, Ilves M, Pösö AR. Sequence variations and two levels of MCT1 and CD147 expression in red blood cells and gluteus muscle of horses. Gene 2012;491:65-70.

[8] Soares OAB, Ferraz GC, Trigo P, D’Angelis FHF, Feringer-Júnior WH, Nardi KB, Almeida FQ, Queiroz Neto A. Comparison between specific and nonspecific tests for evaluating the physical fitness of show jumping horses. Comp Exerc Physiol 2016;12:131-40.

[9] Feringer-Junior WH, Carvalho JRG, Almeida MLM, Queiroz-Neto A, Ferraz GC. Lactate transport in red blood cells by monocarboxylate transporter MCT1 and its accessory protein CD147 in Brazilian sport horses of different performance levels. Equine Vet J Suppl 2014;46:17.

[10] Takimoto M, Takeyama M, Hamada T. Possible involvement of AMPK in acute exercise-induced expression of monocarboxylate transporters MCT1 and MCT4 mRNA in fast-twitch skeletal muscle. Metabolism 2013;62:1633-40.

[11] Väihkönen LK, Ojala M, Pösö AR. Age-related changes and inheritance of lactate transport activity in red blood cells. Equine Vet J Suppl 2002;3:568-72.

[12] Who's Certified [internet]. Tonsel. Horse Telex BV, http://www. horsetelex.com/horses/semen; [accessed 16.05.2016].

[13] Personal communication by Adriana Busato. Curitiba, Brazil: Pontifical Catholic University of Paraná; 2016.
[14] Koenen EPC, Aldridgeb LI, Philipsson J. An overview of breeding objectives for warmblood sport horses. Livest Prod Sci 2004;88:77-84.

[15] Thorén Hellsten E, Jorjani H, Philipsson J. Connectedness among five European sport horse populations. Livest Sci 2008;118:147-56.

[16] Ruhlmann C, Bruns E, Fraehr E, Philipsson J, Janssens S, Quinn K, et al. Genetic connectedness between seven European countries for performance in jumping competitions of warmblood riding horses. Livest Sci 2009;120:75-86.

[17] Dias MG, Bergmann JAG, Rezende ACCG, Castro HF. Formação estrutura populacional do equino Brasileiro de Hipismo. Arq Bras Med Vet Zoo 2000;52:647-54. Portuguese.

[18] Mykkänen A. Expression of lactate transporters MCT1, MCT2, MCT4 and the ancillary protein CD147 in horse muscle and red blood cells [Dissertation]. Helda, University of Helsinki; 2011. Available at: http://ethesis.helsinki.fi.

[19] Bower MA, McGivney BA, Campana MG, Gu J, Andersson LS Barrett $\mathrm{E}$, et al. The genetic origin and history of speed in the Thoroughbred racehorse. Nat Commun 2012;643:1-8.

[20] Regatieri IC, Almeida MLM, Neto ART, Curi RA, Ferraz GC, QueirozNeto A. Quantification of MCT1 and CD147 in red blood cells of Arabian and Quarter Horses. J Equine Vet Sci 2016;43:66-71.

[21] Piccione G, Messina V, Casella S, Giannetto C, Caola G. Blood lactate levels during exercise in athletic horses. Comp Clin Path 2010;19 535-9.

[22] Art T, Amory H, Desmecht D, Lekeux P. Effect of show jumping on heart rate, blood lactate and other plasma biochemical values. Equine Vet J 1990;9:78-82.

[23] Evans DL, Harrls RC, Snow DH. Correlation of racing performance with blood lactate and heart rate after exercise in Thoroughbred horses. Equine Vet J 1993:25:441-5.

[24] Juel C, Lundby C, Sander M, Calbet JA, Hall GV. Human skeleta muscle and erythrocyte proteins involved in acid-base homeostasis: adaptations to chronic hypoxia. J Physiol 2003;15:639-48.

[25] Kitaoka Y, Masuda H, Mukai K, Hiraga A, Takemasa T, Hatta H. Effect of training and detraining on monocarboxylate transporter (MCT) 1 and MCT4 in Thoroughbred horses. Exp Physiol 2011;96:348-55.

[26] Cottin F, Metayer N, Goachet AG, Julliand V, Slawinski J, Billat V, Barrey E. Oxygen consumption and gait variables of Arabian endurance horses measured during a field exercise test. Equine Vet J 2010;38:1-5.

[27] McKenzie E, Marlin D. Science-in-brief: The 9th Meeting of the International Conference on Equine Exercise Physiology 2014. Biochemistry, nutrition and cardiorespiratory function of athletic horses. Equine Vet J 2015;47:254-6. 